\title{
Versatile peptide rafts for conjugate morphologies by self-assembling amphiphilic helical peptides
}

\begin{abstract}
Motoki Ueda $^{1}$, Akira Makino ${ }^{1}$, Tomoya Imai $^{2}$, Junji Sugiyama ${ }^{2}$ and Shunsaku Kimura ${ }^{1}$
Amphiphilic helical peptides with opposite helicities and different hydrophobic helical block lengths were combined to prepare self-assemblies in water for constructing novel morphologies. (Sar) ${ }_{25}-b$-(L-Leu-Aib) 6 and (Sar) $25^{-} b$-(D-Leu-Aib) 6 (sarcosine (Sar) chain, a hydrophobic helix of leucine (Leu) and $\alpha$-aminoisobutyric acid (Aib)) have been reported to self-assemble into welldefined nanotubes, but a 1:1 mixture of these components self-assembles into vesicles. Their mixtures at molar ratios 8:2 and 2:8 have yielded round-bottom flask-shaped molecular assemblies, which are an AB-type conjugate morphology of a vesicle (A) and a nanotube (B). Here, we expand the conjugate morphologies to new AB-type, ABA-type, $A B A^{\prime}$-type and $A_{3} B$-type. The new $A B$-type conjugate morphology had a nano-test-tube shape. Two types of the nano-test-tube-shaped self-assemblies were prepared using different combinations of nanotubes and vesicles. One thin nanotube was sealed with vesicles at both open ends to generate the ABA-type conjugate morphology with a dumbbell shape. When the round-bottom flask-shaped selfassembly was combined with a small vesicle, the $A B A^{\prime}$-type conjugate morphology was obtained. The combination of a threeneck nanotube with a vesicle produced the $A_{3} B$-type morphology. These self-assemblies were thermodynamically stable owing to the phase separation in their fused membranes. Therefore, these self-assemblies are termed 'peptide rafts' as an analogy to 'lipid rafts'.
\end{abstract}

Polymer Journal (2013) 45, 509-515; doi:10.1038/pj.2013.4; published online 27 February 2013

Keywords: amphiphiles; morphologies; nanotubes; peptides; phase separation; self-assembly; vesicles

\section{INTRODUCTION}

The heterogeneity of cell membranes is considered essential for cell function, and lipid rafts are one origin of the heterogeneity in a membrane structure. In lipid rafts, sphingolipids and cholesterol provide environments in which membrane proteins can function, resulting in a phase separation in the membranes to maintain their fluid properties. ${ }^{1-6}$ Using the phase separation property of lipid rafts, a large lateral separation into two domains with cholesterol/ sphingomyelin/saturated phospholipids and giant unilamellar vesicles of unsaturated phospholipids was demonstrated and used to generate Janus vesicles with a two-vesicle conjugate morphology (AA'-type conjugate morphology). ${ }^{7-9}$ Another reported conjugate morphology of self-assemblies was a round-bottom flask-type morphology (that is, the AB-type conjugate morphology, where A and $\mathrm{B}$ represent vesicle shape and tube shape, respectively) generated by capping one end of a nanotube with a vesicle, which was prepared from a mixture of phospholipids and amphiphilic molecules with two hydrophobic legs. ${ }^{10,11}$

Apart from phospholipids and lipid-like molecules, amphiphilic helical peptides have been shown to self-assemble into a roundbottom flask-type conjugate morphology. Helical peptides have a good regular packing ability in the molecular assembly as shown by frequent observations of helix bundles in proteins. ${ }^{12,13}$ Several hydrophobic helical peptides with suitable hydrophilic groups attached have formed vesicle assemblies with diameters of approximately $100 \mathrm{~nm}$ in water, which are termed 'peptosomes.' ${ }^{14}$ Furthermore, a peptide nanotube with a diameter of approximately $70 \mathrm{~nm}$ and a length of approximately $200 \mathrm{~nm}$ was obtained from an amphiphilic block polypeptide composed of a hydrophilic sarcosine (Sar) chain, a hydrophobic helix of leucine (Leu) and $\alpha$-aminoisobutyric acid (Aib), (Sar) ${ }_{25}$ - $b$-(L-Leu-Aib) ${ }_{6}\left(\right.$ S25L12). ${ }^{15}$ This block polypeptide initially formed a curved square sheet assembly, which was transformed into a nanotube morphology upon heating the dispersion at $90{ }^{\circ} \mathrm{C}$ for $10 \mathrm{~min}$. Conversely, a mixture of right- and lefthanded leucine helices formed stereocomplexes with a planar sheet morphology that transformed into a vesicular morphology upon heating. ${ }^{7,8}$ Furthermore, mixtures of the helices at a molar ratio of $8: 2$ or $2: 8$ yielded molecular assemblies with a round-bottom flaskshaped morphology by lateral separation, which was due to selective association of the helices induced by the stereocomplex formation of right- and left-handed helices. ${ }^{15-17}$

In this study, we show that the dimensions of a round-bottom flask-shaped morphology, which is composed of a neck (nanotube origin) and a round-bottom part (vesicle origin), are tunable by

${ }^{1}$ Department of Material Chemistry, Graduate School of Engineering, Kyoto University, Kyoto-Daigaku-Katsura, Nishikyo-ku, Kyoto, Japan and ${ }^{2}$ Research Institute for Sustainable Humanosphere (RISH), Kyoto University, Gokasho, Uji, Kyoto, Japan

Correspondence: Professor S Kimura, Department of Material Chemistry, Graduate School of Engineering, Kyoto University, Kyoto-Daigaku-Katsura, Nishikyo-ku, Kyoto 615-8510, Japan.

E-mail: shun@scl.kyoto-u.ac.jp

Received 30 September 2012; revised 18 November 2012; accepted 19 November 2012; published online 27 February 2013 

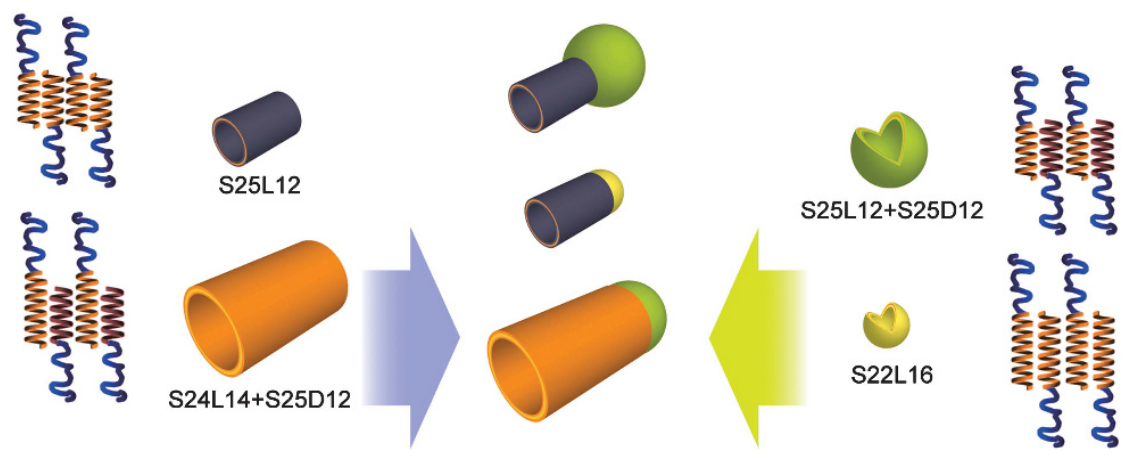

Figure 1 Schematic of conjugate morphology self-assemblies composed of size-controlled nanotubes and vesicles. Amphiphilic helical block peptides form tubular and vesicular structures. By incubating and heat treating the nanotubes and vesicles, vesicles are capped onto the open end of the nanotubes, resulting in the formation of round-bottom- and test-tube-shaped assemblies due to phase separation in the peptide membranes. This strategy is applicable to various-sized nanotubes and vesicles and leads to size-controlled conjugate morphologies.

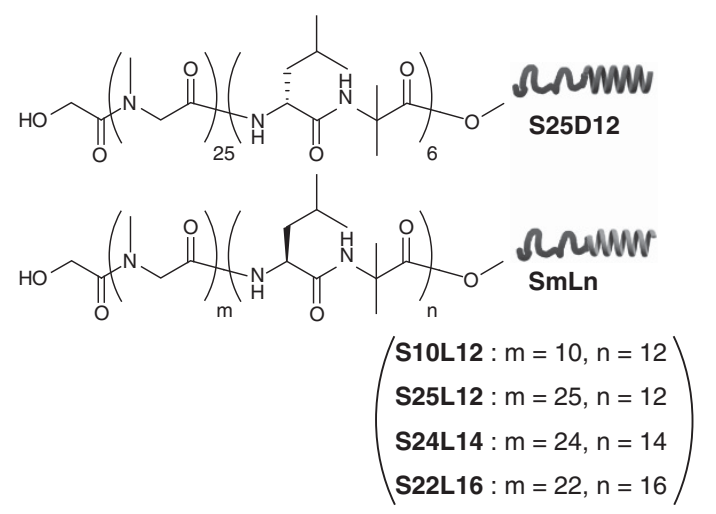

Figure 2 Chemical structures of amphiphilic block polypeptides S25D12, S10L12, S25L12, S24L14 and S22L16. A full color version of this figure is available at Polymer Journal online.

changing the combination of the amphiphilic helical peptides (Figure 1). In addition, an advanced $\mathrm{ABA}^{\prime}$-type conjugate morphology with a nanotube and two vesicles can be prepared by the lateral phase separation of self-assembled membranes composed of three types of amphiphilic helical peptides.

\section{EXPERIMENTAL PROCEDURES}

\section{Amphiphilic helical peptides}

Amphiphilic polypeptides of $\mathrm{Sar}_{25}-b$-(D-Leu-Aib) 6 (S25D12), $\mathrm{Sar}_{n}-b$ -

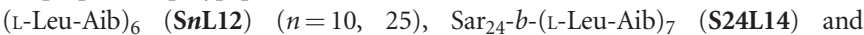
$\mathrm{Sar}_{22}-b$-(L-Leu-Aib) 8 (S22L16) (Figure 2) were synthesized as reported previously. ${ }^{12,14}$ The syntheses of all compounds were confirmed by proton nuclear magnetic resonance spectroscopy experiments and matrix-assisted laser desorption/ionization-time of flight mass spectrometry.

\section{Preparation of molecular assemblies}

The polypeptide $(12 \mathrm{mg})$ was dissolved in ethanol $(240 \mu \mathrm{l})$ to make a stock solution. An aliquot $(10 \mu \mathrm{l})$ of the stock solution was injected into a buffer $(10 \mathrm{~mm}$ Tris- $\mathrm{HCl}, \mathrm{pH} 7.4)(1 \mathrm{ml})$ while stirring at $4{ }^{\circ} \mathrm{C}$. After stirring for $30 \mathrm{~min}$, this dispersion was heated at $90^{\circ} \mathrm{C}$ for $1 \mathrm{~h}$. Molecular assemblies with different compositions were prepared using a similar method. For compositions with multiple polypeptides, the stock ethanol solutions of the amphiphilic polypeptides were mixed to obtain $10 \mu \mathrm{l}$ of the mixture with the desired ratio before injection into the buffer. The mixture solution was then injected and heated in the same manner.

\section{Transmission electron microscopy}

Transmission electron microscopy images were taken using a JEOL JEM2000EXII electron microscope (JEOL, Tokyo, Japan) with an accelerating voltage of $100 \mathrm{kV}$. A drop of each dispersion was mounted on a carbon-coated $\mathrm{Cu}$ grid and negatively stained with $2 \%$ uranyl acetate, followed by suctioning off the excess fluid using a filter paper.

\section{Frozen-hydrated/cryogenic-TEM}

The dispersions in the buffer were quickly frozen in liquid ethane, which was cooled with liquid nitrogen. The samples were examined using a $100 \mathrm{kV}$ accelerating voltage at the liquid nitrogen temperature.

\section{RESULTS AND DISCUSSION}

\section{Size control of the sphere part of a conjugate morphology}

S22L16 (Figure 2) self-assembled into vesicles with approximately $70 \mathrm{~nm}$ diameters in the buffer upon heating the planar sheets of S22L16, which were instantly formed using the injection method (Figures $3 \mathrm{a}-\mathrm{c}$ and Supplementary Figure $\mathrm{S} 1$ in Supplementary information (SI)), at $90^{\circ} \mathrm{C}$ for $1 \mathrm{~h}$. This vesicle diameter is similar to the diameter of $\mathbf{S 2 5 \mathrm { L } 1 2}$ nanotubes, leading to the idea of combining S22L16 and S25L12 to obtain an AB-type conjugate morphology with a round-bottom test tube. Accordingly, S22L16 planar sheets were incubated with S25L12 nanotubes at a ratio of 0.5:1 (v/v), and the dispersion was heated at $90^{\circ} \mathrm{C}$ for $1 \mathrm{~h}$. As shown in Figures $3 \mathrm{~d}$ and e, nano-test-tube-shaped self-assemblies with round bottoms were predominantly observed by TEM. The sizes of the neck and round-bottom parts of the assemblies correspond to those of the S25L12 nanotube and S22L16 vesicle, respectively. Furthermore, the membrane thickness of the spherical part is $6 \mathrm{~nm}$, which is the same as that of the S22L16 vesicle (Figure 3g). These results suggest that the nano-test-tube-shaped self-assembly is composed of a peptide membrane where S25L12 and S22L16 phase separated to form the corresponding neck part and spherical part of the nano-test-tube morphology. By increasing the mixing ratio of S22L16 (vesicle component) to S25L12 (nanotube component) from $0.5: 1-1: 1$ to 2:1 (v/v), the yields of the ABA-type nanocapsules, where both open ends of the S25L12 nanotube were sealed with round-bottom-shaped S22L16 membranes (Figure $3 f$ and Supplementary Figure S2), increased drastically from 11 to 36 to $67 \%$ (Figure $3 \mathrm{~h}$ ). The AB-type nano-test-tube morphology or the ABA-type nanocapsule morphology could therefore be prepared as a certain fraction of the mixture by optimizing the mixing ratio of the nanotube to the planar sheet. 

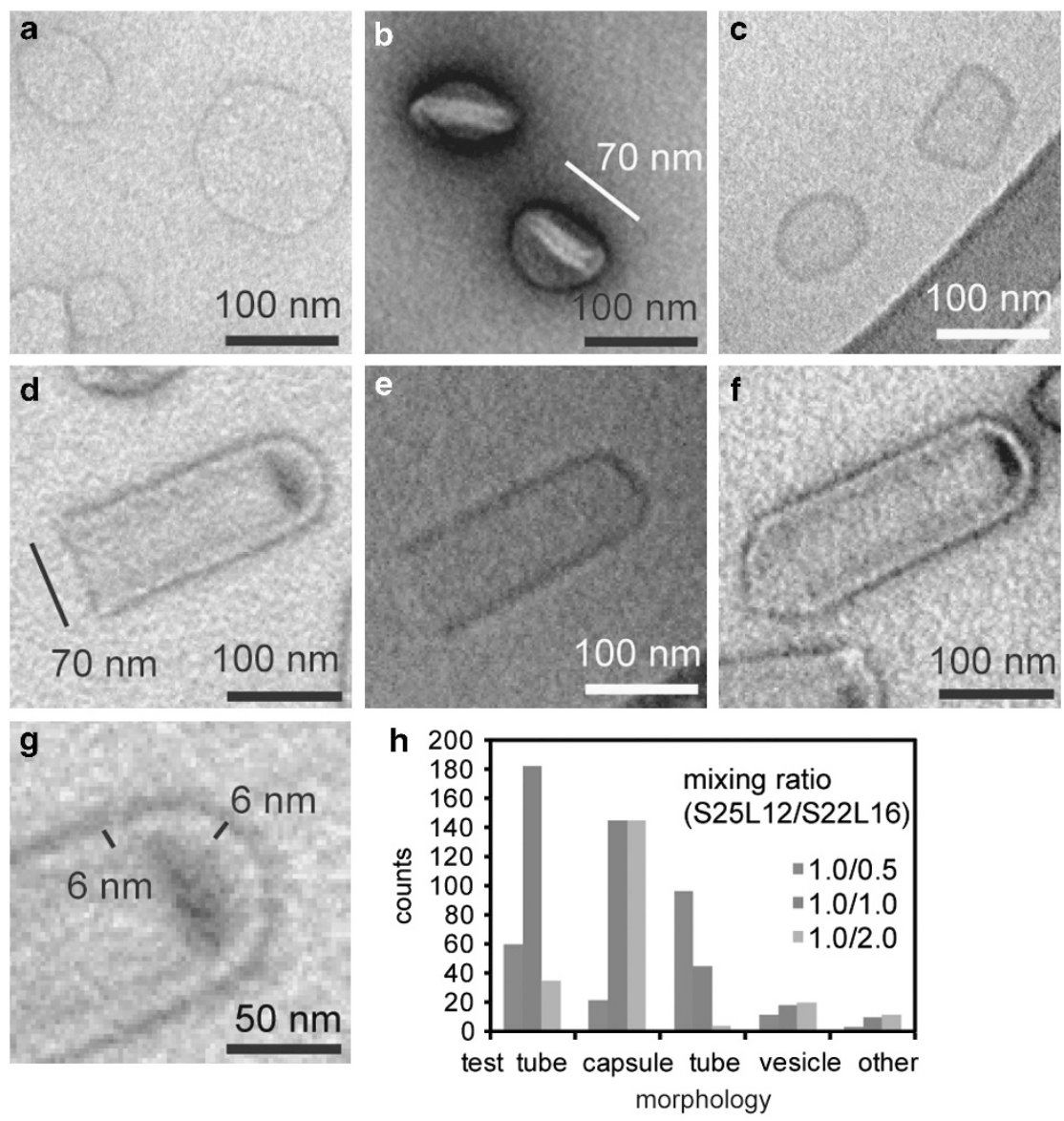

Figure 3 Transmission electron microscopy (TEM) images (negative staining with uranyl acetate (a, b, $\mathbf{d}$ and f); cryogenic TEM (c and e)) of molecular assemblies prepared from the single component of S22L16 (a-c) and mixtures of constituent assemblies (S25L12 nanotube and S22L16 sheet) (d-f). The TEM micrograph ( $\mathbf{g}$ ) is the magnification of micrograph (d). The histogram (h) shows the product quantity for each type of morphology found in the sample dispersions using TEM. A full color version of this figure is available at Polymer Journal online.

The phase separation into the tube domain of S25L12 and the round domain of S22L16 in the nano-test-tube-type and nanocapsule-type morphologies was maintained when heating these morphologies at $90^{\circ} \mathrm{C}$ for $1 \mathrm{~h}$, suggesting that they are thermodynamically stable. This observation is consistent with our previous report that S25L12 nanotubes could not fuse with S22L16 vesicles, suggesting that the molecular miscibility of S25L12 and S22L16 is low after forming the corresponding morphologies even with a heat treatment at $90^{\circ} \mathrm{C} .18$ Two types of S25L12 and S25D12 nanotubes were found to fuse with each other because the stereocomplex formation of S25L12 and S25D12 promotes their mixing, leading to planar sheets even at room temperature. ${ }^{16}$ Pairing the right- and left-handed helices is thus the key factor to mixing the two corresponding phases.

Instead of starting with the nanotube and planar sheet, an ethanol solution of a S25L12 and S22L16 mixture $(1: 1(\mathrm{w} / \mathrm{w}))$ was injected directly into the buffer, followed by a heat treatment at $90^{\circ} \mathrm{C}$ for $1 \mathrm{~h}$. As determined by TEM, the nano-test-tube-type and nanocapsuletype self-assemblies were similar to those obtained from mixing the nanotube with the planar sheet. The shapes and sizes of the nanotest-tube-type and nanocapsule-type self-assemblies are therefore independent of these two preparation methods, further indicating that these conjugate morphologies are thermodynamically determined. In fact, based on TEM observations, these molecular assemblies retained their morphologies for at least 1 month.
Size control of the neck part of a conjugate morphology

The use of other combinations of nanotubes and vesicles for preparing the nano-test-tube-type conjugate morphology is examined. A vesicle $180 \mathrm{~nm}$ in diameter was prepared from an equimolar mixture of S25L12 and S25D12, and a thick nanotube $200 \mathrm{~nm}$ in diameter and $500 \mathrm{~nm}$ in length was prepared from an equimolar mixture of S25D12 and S24L14 (Figures 4a and b and Supplementary Figure S3). ${ }^{19}$ When the thick nanotubes were incubated with the planar sheets prepared from S25L12 and S25D12 and heated at $90^{\circ} \mathrm{C}$ for $1 \mathrm{~h}$, round-bottom test-tube-shaped self-assemblies were generated with a yield of $78 \%$ ((round-bottom test tube $(n=63)) /($ roundbottom test tube + test tube $(n=81))$ ) (Figure $4 \mathrm{f}$ ), where the neck and round-bottom parts have the same dimensions as the corresponding S25D12 and S24L14 nanotube and S25L12 and S25D12 vesicle (Figures $4 \mathrm{c}-\mathrm{e}$ ). This nano-test-tube-shaped morphology is therefore reasonably explained by the planar sheet sticking to one open end of the nanotube and then transforming into a spherical membrane to seal the mouth of the nanotube shut. Thin and thick nano-test-tube-shaped morphologies can therefore be prepared by choosing different combinations of amphiphilic helical peptides.

Once the thick nano-test-tube-shaped self-assembly was formed, the morphology was preserved for a day even at $90^{\circ} \mathrm{C}$, suggesting that the thick nano-test-tube-shaped self-assembly is thermodynamically stable. However, when an ethanol solution of a mixture of S25D12, 

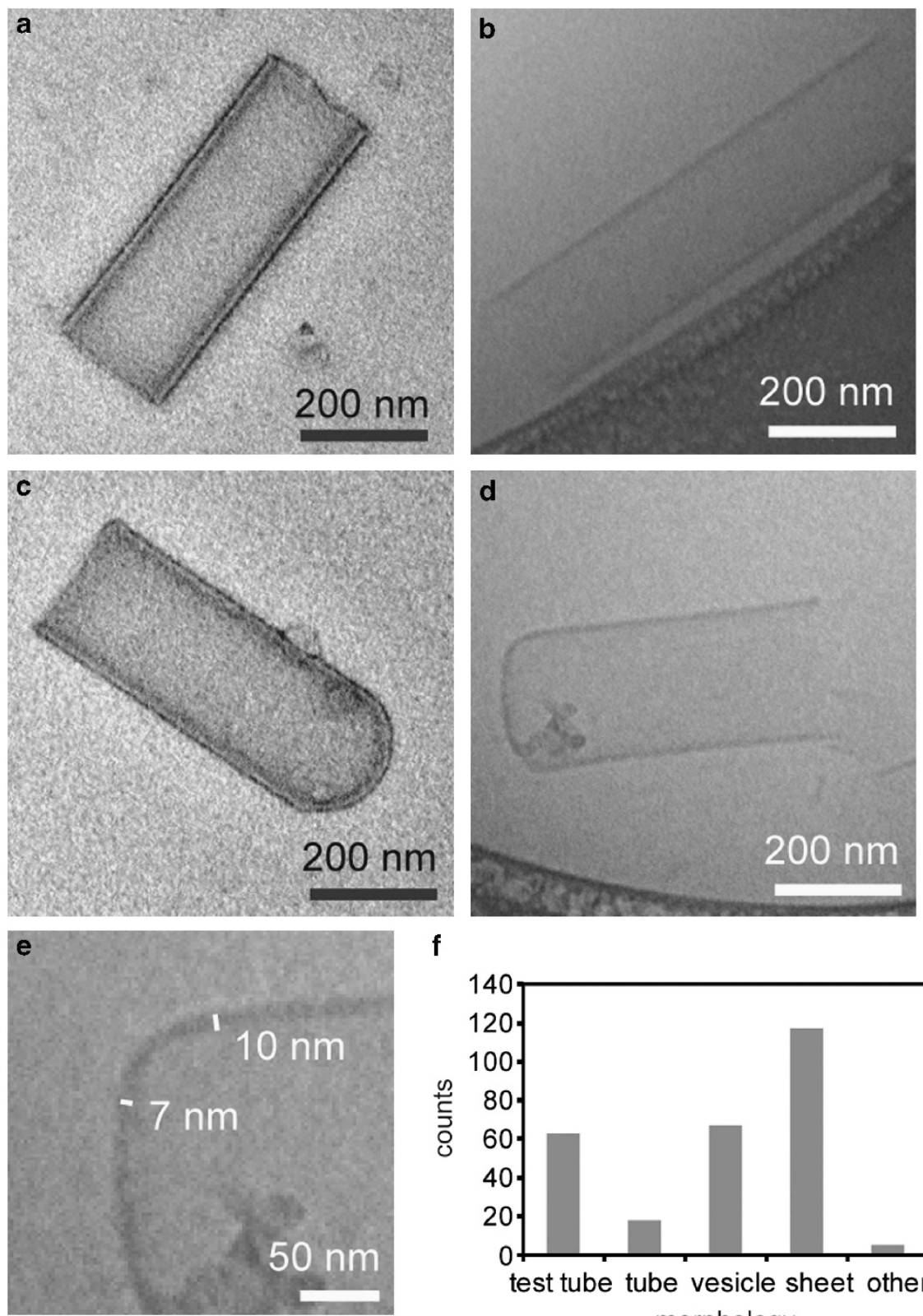

f

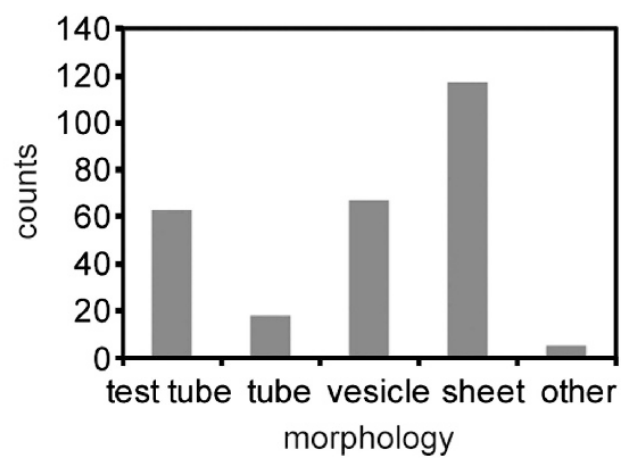

Figure 4 Transmission electron microscopy (TEM) images (negative staining with uranyl acetate (a and c); cryogenic TEM (b, d and e)) of molecular assemblies prepared from mixtures of constituent assemblies (S25D12 + S24L14 nanotube and S25L12 + S25D12 sheet). The TEM micrograph (e) is the magnification of micrograph (d). The histogram (f) shows the product quantity for each type of morphology found in the sample dispersions using TEM. A full color version of this figure is available at Polymer Journal online.

S25L12 and S24L14 $(2: 1: 1(\mathrm{w} / \mathrm{w} / \mathrm{w}))$ was injected directly into the buffer followed by a heat treatment at $90^{\circ} \mathrm{C}$ for $1 \mathrm{~h}$, vesicles were formed, as observed in the TEM observations (Figure 5). Most likely, the three-component system could induce more phase separation than the two-component system, which would make it difficult for the three components to converge into one specific $\mathrm{AB}$-type conjugate morphology just by injecting their mixture into water.

These two types of nano-test-tube self-assemblies, that is, the small S25L12 and S22L16 one and the large S25D12, S25L12 and 24L14 one, resulted in the hydrophobic edges of the nanotubes sticking to the vesicle-forming sheets, keeping each phase in one fused membrane. In these cases, the pairing stability in the membrane should decrease in the order (S25D12 and S25L12) $>$ (S25D12 and 24L14) $\gg$ (S25L12 and S22L16). There seem to be two factors for each pair of helical peptide types, that is, the stereochemistry and the helix-chain length, that determine their miscibility in the membrane.

\section{More complex conjugate morphologies}

An $\mathrm{ABA}^{\prime}$-type conjugate morphology of two different vesicles and one nanotube was prepared from three types of amphiphilic helical peptides by phase separation (Figure 6a). First, the round-bottom 


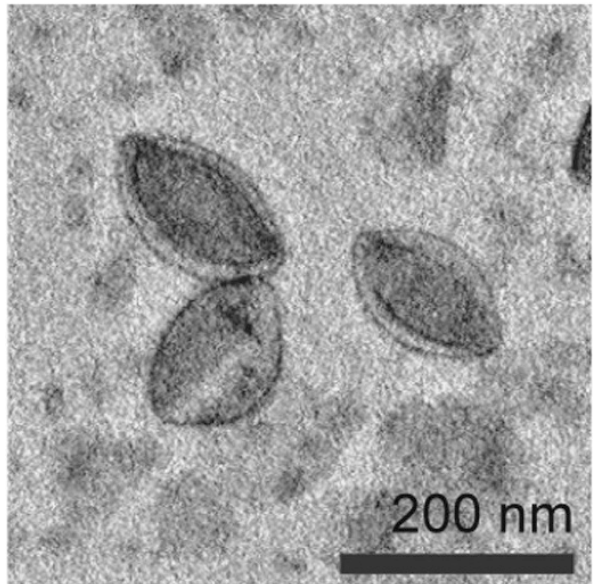

Figure 5 Transmission electron microscopy (TEM) images (negative staining with uranyl acetate) of molecular assemblies prepared from mixtures of constituent assemblies (S25D12 + S25L12 + S24L14, molar ratio 2:1:1) after heat treatment at $90^{\circ} \mathrm{C}$ for $1 \mathrm{~h}$ in $10 \mathrm{~mm}$ Tris- $\mathrm{HCl}$ buffer $(\mathrm{pH} 7.4)$. flask assemblies were prepared from a mixture of S25L12 and S25D12 at the molar ratio $2: 8$ and purified using a syringe filter to remove assemblies larger than $450 \mathrm{~nm}$. Subsequently, S22L16 planar sheets were added, and the mixture was heated at $90^{\circ} \mathrm{C}$ for $1 \mathrm{~h}$. Using this two-step method, $\mathrm{ABA}^{\prime}$-type asymmetric dumbbell-shaped assemblies were obtained with a yield of 36\% ((dumbbell)/(dumbbell + roundbottom flask)) (Figures $6 \mathrm{~b}-\mathrm{d}$ and Supplementary Figure S4). As shown in Figure 6b, the asymmetric dumbbell-shaped morphology has two different spherical parts that are each 180 and $70 \mathrm{~nm}$ in diameter and a middle neck part that is $70 \mathrm{~nm}$ in diameter and $220 \mathrm{~nm}$ in length, which correspond to the stereocomplex vesicle composed of S25L12 and S25D12, the S22L16 vesicle and the S25L12 nanotube. An ABA'-type conjugate morphology is therefore attainable by maintaining phase separation in one peptide membrane. The histogram in Figure 6e shows the product quantity for each type of morphology found in the sample dispersions using TEM, indicating that the yield of the dumbbell-shaped assemblies was $8 \%$.

Three-way nanotubes were combined with vesicles to generate an $\mathrm{A}_{3} \mathrm{~B}$-type conjugate morphology (Figure 7a). The three-way
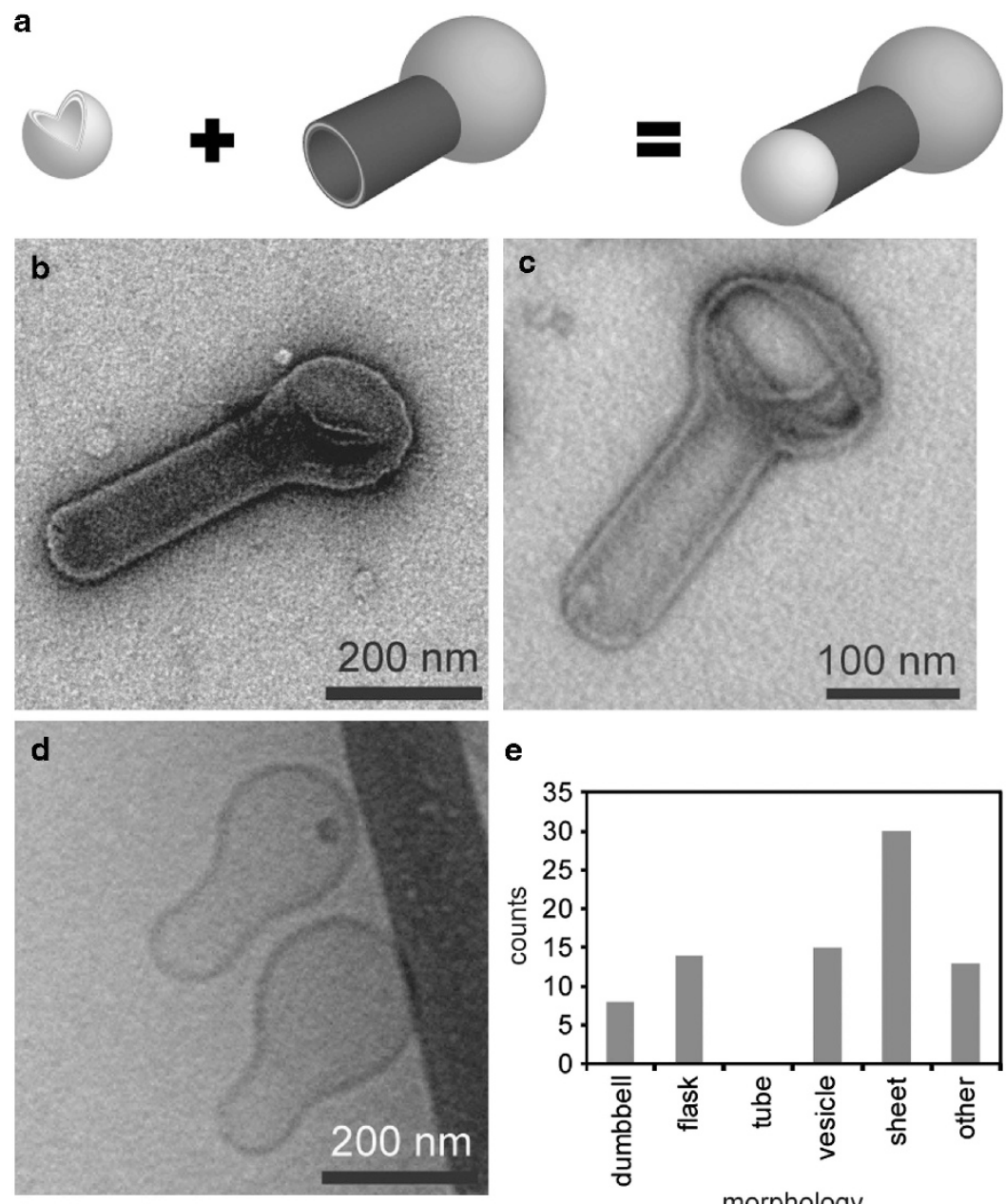

e

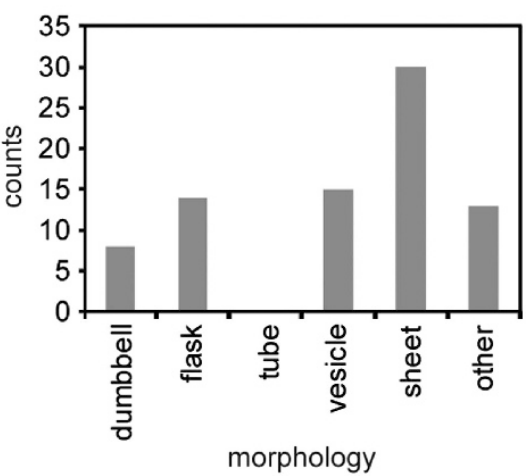

Figure 6 Illustrations of phase-separated self-assemblies composed of three different membranes (a). Transmission electron microscopy (TEM) images (negative staining with uranyl acetate (b and $\mathbf{c}$ ); cryogenic TEM (d)) of molecular assemblies prepared from mixtures of constituent assemblies (S25L12 + S25D12 round-bottom flask and S22L16 sheet (b-d)). The dumbbell-type conjugate morphology was prepared by injecting the S22L16 planar sheet dispersion into the pre-prepared round-bottom flask dispersion, which was composed of S25L12 + S25D12 vesicles and S25D12 nanotubes, and heat treating the mixture at $90^{\circ} \mathrm{C}$ for $10 \mathrm{~min}$. The histogram (e) shows the product quantity for each type of morphology found in the sample dispersions using TEM. A full color version of this figure is available at Polymer Journal online. 
a

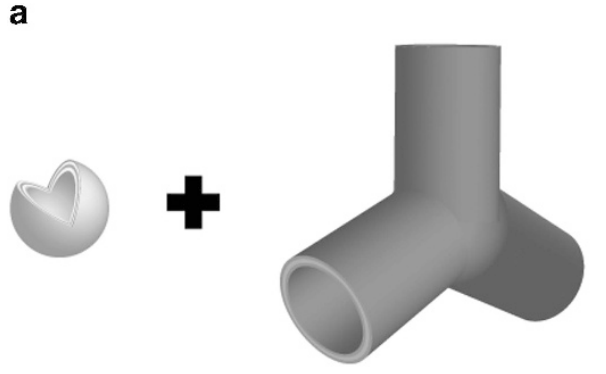

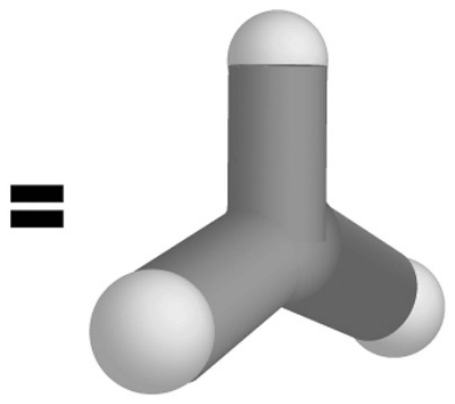
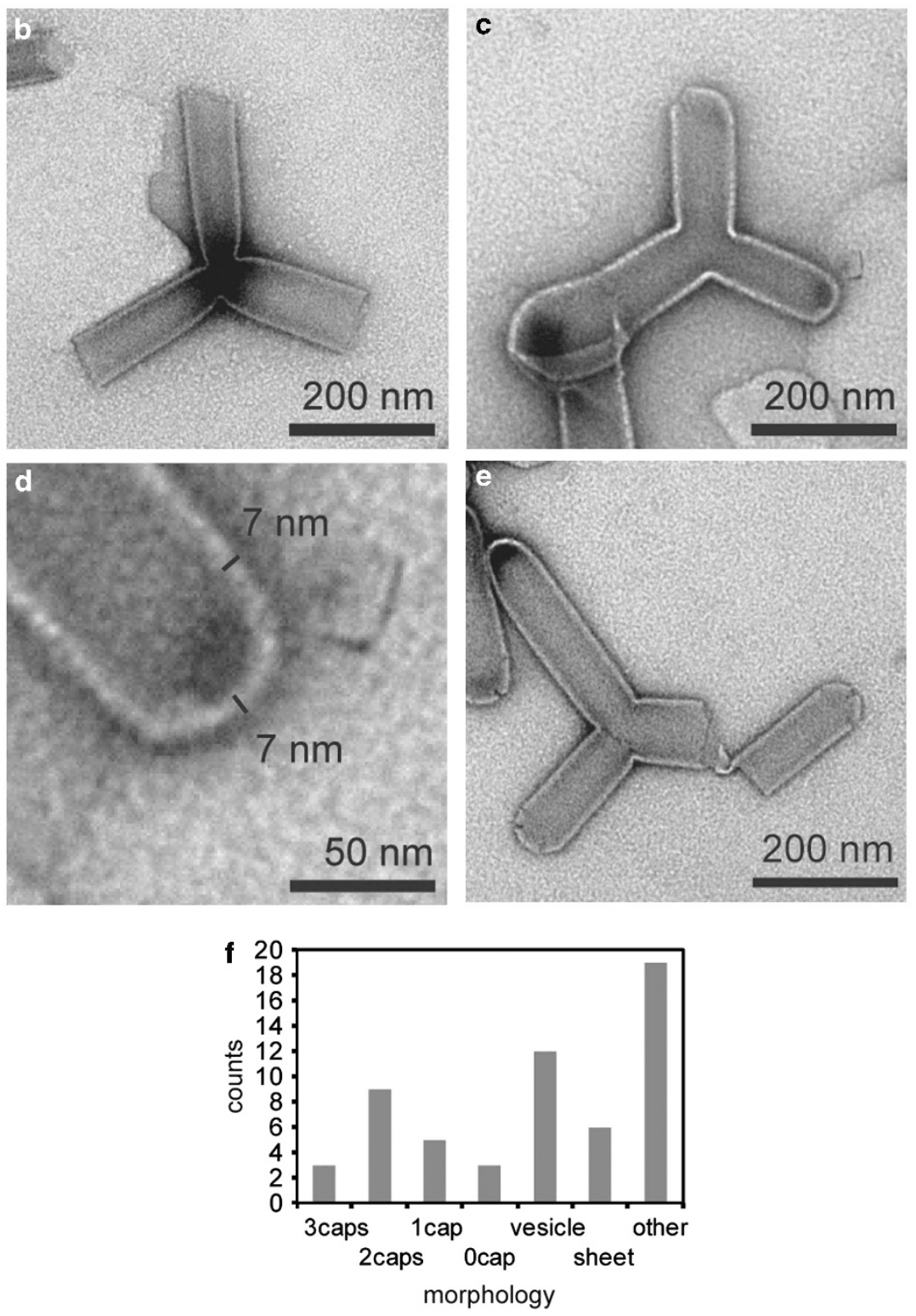

Figure 7 Illustrations of phase-separated self-assemblies composed of a three-way tube and vesicles (a). Transmission electron microscopy (TEM) images (negative staining with uranyl acetate $(\mathbf{b}-\mathbf{e})$ ) of molecular assemblies prepared from mixtures of constituent assemblies (S25L12 + S10L12 three-way nanotube and S22L16 sheet (b-e)). The TEM micrograph (d) is the magnification of micrograph (c). The vesicle-capped three-way nanotube was prepared by mixing the three-way nanotube dispersion and planar sheet dispersion and heating the mixture at $90^{\circ} \mathrm{C}$ for $1 \mathrm{~h}$. The histogram (f) shows the product quantity for each type of morphology found in the sample dispersions using TEM. A full color version of this figure is available at Polymer Journal online. 
nanotubes, which were prepared from an equimolar mixture of S25L12 and S10L12 (Figure 7b), ${ }^{15}$ were incubated with S22L16 sheets at the molar ratio 1:3 ((S25L12 + S10L12)/S22L16) and heated at $90{ }^{\circ} \mathrm{C}$ for $1 \mathrm{~h}$. As shown in Figure $7 \mathrm{c}$, the $\mathrm{A}_{3} \mathrm{~B}$-type three-way nanocapsules were observed by TEM. However, the yield of $\mathrm{A}_{3} \mathrm{~B}$-type nanocapsules did not exceed 5\% (Figure 7f) because three-way nanotubes with one end capped and two ends capped with vesicles were mixed with the $\mathrm{A}_{3} \mathrm{~B}$-type nanocapsules (Figure 7e). The dimensions of the neck part and spherical part of the conjugate morphologies were consistent with those of the nanotubes and vesicles, respectively (Figure 7d), suggesting that S22L16 selfassembled onto the open ends of the three-way nanotubes to form the spherical parts, keeping the phase separation in the fused membrane.

\section{CONCLUSIONS}

All of these examples of AB-type, $A B A$-type, $\mathrm{ABA}^{\prime}$-type and $\mathrm{A}_{3} \mathrm{~B}$-type conjugate morphologies were prepared using suitable combinations of amphiphilic helical peptides. The hydrophobic core regions of the peptide membranes were formed by side-by-side associations of the helices. The properties of these associations should be determined by the stereochemistry and helix-chain length, as shown here. Other expected association factors, such as the concave-convex surface fitness between helices (primary sequence), dipole-dipole interaction and helix type ( $\alpha-, 3_{10}, \beta-$, double, triple), remain to be investigated. 'Peptide rafts', which are associated with helices, will therefore be more versatile than lipid rafts in creating conjugate morphologies by self-assembly and lateral phase separation in membranes.

\section{ACKNOWLEDGEMENTS}

This study is part of a joint research venture, which focuses on the development of a technological basis to establish COE for nanomedicine, conducted through the Kyoto City Collaboration of Regional Entities for Advancing Technology Excellence (CREATE) assigned by the Japan Science and Technology Agency (JST).
1 Anderson, R. G. W. \& Jacobson, K. Cell biology - a role for lipid shells in targeting proteins to caveolae, rafts, and other lipid domains. Science 296, 1821-1825 (2002).

2 Simons, K. \& Gruenberg, J. Jamming the endosomal system: lipid rafts and lysosomal storage diseases. Trends Cell. Biol. 10, 459-462 (2000).

3 Simons, K. \& Toomre, D. Lipid rafts and signal transduction. Nat. Rev. Mol. Cell. Biol. 1, 31-39 (2000).

4 Bagnat, M., Keranen, S., Shevchenko, A., Shevchenko, A. \& Simons, K. Lipid rafts function in biosynthetic delivery of proteins to the cell surface in yeast. Proc. Natl. Acad. Sci. USA 97, 3254-3259 (2000).

5 Brown, D. A. \& London, E. Structure and function of sphingolipid- and cholesterol-rich membrane rafts. J. Biol. Chem. 275, 17221-17224 (2000).

6 Simons, K. \& Ikonen, E. Functional rafts in cell membranes. Nature 387, 569-572 (1997).

7 Loew, M., Springer, R., Scolari, S., Altenbrunn, F., Seitz, O., Liebscher, J., Huster, D., Herrmann, A. \& Arbuzova, A. Lipid domain specific recruitment of lipophilic nucleic acids: a key for switchable functionalization of membranes. J. Am. Chem. Soc. 132, 16066-16072 (2010).

8 Christian, D. A., Tian, A. W., Ellenbroek, W. G., Levental, I., Rajagopal, K., Janmey, P. A., Liu, A. J., Baumgart, T. \& Discher, D. E. Spotted vesicles, striped micelles and Janus assemblies induced by ligand binding. Nat. Mater. 8, 843-849 (2009).

9 Semrau, S. \& Schmidt, T. Membrane heterogeneity-from lipid domains to curvature effects. Soft Matter 5, 3174-3186 (2009).

10 Coleman, A. C., Beierle, J. M., Stuart, M. C. A., Macia, B., Caroli, G., Mika, J. T., van Dijken, D. J., Chen, J. W., Browne, W. R. \& Feringa, B. L. Light-induced disassembly of self-assembled vesicle-capped nanotubes observed in real time. Nat. Nanotechnol. 6, 547-552 (2011).

11 Roux, A., Cuvelier, D., Nassoy, P., Prost, J., Bassereau, P. \& Goud, B. Role of curvature and phase transition in lipid sorting and fission of membrane tubules. EMBO J. 24 , 1537-1545 (2005).

12 Milburn, M. V., Prive, G. G., Milligan, D. L., Scott, W. G., Yeh, J., Jancarik, J., Koshland, D. E. \& Kim, S. H. 3-Dimensional structures of the ligand-binding domain of the bacterial aspartate receptor with and without a ligand. Science 254, 1342-1347 (1991).

13 Parker, M. W., Pattus, F., Tucker, A. D. \& Tsernoglou, D. Structure of the membranepore-forming fragment of colicin-A. Nature 337, 93-96 (1989).

14 Kimura, S., Sugiyama, J., Muraji, Y., Kim, D.-H. \& Imanishi, Y. Peptosome: vesicle structure by helical peptide monolayer. Peptide Sci. 2000, 397-400 (2001).

15 Kanzaki, T., Horikawa, Y., Makino, A., Sugiyama, J. \& Kimura, S. Nanotube and threeway nanotube formation with nonionic amphiphilic block peptides. Macromol. Biosci. 8, 1026-1033 (2008)

16 Ueda, M., Makino, A., Imai, T., Sugiyama, J. \& Kimura, S. Transformation of peptide nanotubes into a vesicle via fusion driven by stereo-complex formation. Chem. Commun. 47, 3204-3206 (2011).

17 Ueda, M., Makino, A., Imai, T., Sugiyama, J. \& Kimura, S. Tubulation on peptide vesicles by phase-separation of a binary mixture of amphiphilic right-handed and lefthanded helical peptides. Soft Matter 7, 4143-4146 (2011).

18 Ueda, M., Makino, A., Imai, T., Sugiyama, J. \& Kimura, S. Temperature-triggered fusion of vesicles composed of right-handed and left-handed amphiphilic helical peptides. Langmuir 27, 4300-4304 (2011).

19 Ueda, M., Makino, A., Imai, T., Sugiyama, J. \& Kimura, S. Rational design of peptide nanotubes for varying diameters and lengths. J. Pept. Sci. 17, 94-99 (2011).

Supplementary Information accompanies the paper on Polymer Journal website (http://www.nature.com/pj) 